\title{
Coulisses
}

Revue de théâtre

7| Printemps 1993

Varia

\section{Voyage au bout de l'ennui : Léonce et Léna de Georg Büchner}

Gérard Jugan

\section{OpenEdition}

1 Journals

Édition électronique

URL : http://journals.openedition.org/coulisses/2207

DOI : $10.4000 /$ coulisses.2207

ISSN : 2546-9460

Éditeur

Presses universitaires de Franche-Comté

\section{Édition imprimée}

Date de publication : 1 avril 1993

Pagination : 22-28

ISSN : 1150-594X

\section{Référence électronique}

Gérard Jugan, « Voyage au bout de l'ennui : Léonce et Léna de Georg Büchner », Coulisses [En ligne], 7 . Printemps 1993, mis en ligne le 15 mars 2019, consulté le 21 octobre 2019. URL : http:// journals.openedition.org/coulisses/2207; DOI : 10.4000/coulisses.2207

Ce document a été généré automatiquement le 21 octobre 2019.

Coulisses 


\title{
Voyage au bout de l'ennui : Léonce et Léna de Georg Büchner
}

\author{
Gérard Jugan
}

\section{Une drôle de comédie}

1 Léonce et Léna, avec La Mort de Danton et Woyzeck, l'une des trois pièces qui composent l'œuvre dramatique de Georg Büchner, est d'un accès malaisé car elle se dissimule derrière un masque : elle se donne l'allure avenante d'une comédie un peu féerique où le hasard fait trop bien les choses, mais derrière cette façade se précise en réalité au fil des scènes une sensibilité d'une grande richesse et c'est elle qui fait de cette comédie en trompe-l'œil une œuvre profonde et attachante.

2 C'est en effet à première vue une pièce plaisante. Elle raconte avec beaucoup de brio et d'humour comment, dans un minuscule royaume de conte de fées, avec son roi balourd, ses courtisans empressés et des sujets bien dociles, Léonce, jeune prince oisif et désenchanté, s'enfuit pour se soustraire à son existence de roi et, en premier lieu, au mariage qui va l'inaugurer. Il doit épouser la princesse Léna, vouée elle aussi à se marier avec un être qu'elle n'a pas choisi. Valério, compagnon de Léonce, son valet et son mentor à la fois, l'accompagne dans sa fuite. Les deux jeunes gens se rencontrent, s'éprennent l'un de l'autre en ignorant qui ils sont réellement et le périple se termine par un mariage où l'amour rejoint la raison d'Etat.

3 L'intérêt de l'œuvre et sa force ne résident manifestement pas dans cette intrigue qui accumule sans craintes bien des motifs traditionnels: le prince mélancolique et désœuvré que le baiser de la princesse va tirer de son sommeil, la fuite pour échapper l'un à l'autre, et qui conduit l'un à l'autre, le coup de foudre, le départ pour l'Italie, la conclusion heureuse de l'aventure. Beaucoup de convention, d'accessoires de la féerie et du romantisme allemands. Büchner a beaucoup lu, beaucoup admiré et la pièce en porte la marque. ${ }^{1} \mathrm{Et}$ pourtant, malgré cette affabulation fort peu réaliste, Léonce et Léna est une pièce foncièrement réaliste, profondément vraie et vivante parce qu'elle énonce avec une totale sincérité, dans le langage étincelant qui est le sien, le débat, 
bien réel lui, situé au centre de l'existence du très jeune homme qu'est encore Georg Büchner quand il l'écrit : l'angoisse profonde éprouvée par un jeune allemand de 1836 en face de la vie, du temps, de lui-même et aussi de l'Histoire, qui semble, à cette époque de contre-révolution, marquer le pas. Büchner en fera très vite la douloureuse expérience ${ }^{2}$. Cette angoisse, nourrie d'érotisme et de pulsions morbides, imprègne la pièce. Elle chemine, souterraine parfois, d'une scène à l'autre, en dépit des éclats de rire et du merveilleux et c'est elle qu'il faut commencer à cerner si l'on veut comprendre pourquoi Léonce et Léna n'est pas une comédie comme les autres, mais une pièce grave qui nous raconte la difficile guérison d'un être blessé.

\title{
Ma tête est une salle de danse abandonnée
}

\begin{abstract}
LÉONCE, seul (...)
Ma vie me regarde en bâillant comme une grande main de papier blanc que je devrais couvrir de mon écriture, mais je suis incapable de former une seule lettre. Ma tête est une salle de danse abandonnée, avec quelques roses fanées et des rubans froissés par terre, il y a des violons crevés dans un coin, les derniers danseurs ont enlevé leurs masques et se regardent avec des yeux emplis d'une fatigue mortelle. Je me retourne comme un gant vingt-quatre fois dans la journée. Oh! je me connais. Je sais ce que je penserai, ce que je rêverai dans un quart d'heure, dans huit jours, dans un an. Dieu, quel crime ai-je donc commis pour que tu me fasses réciter ma leçon aussi souvent qu'à un écolier ? ${ }^{3}$
\end{abstract}

4 Cette cascade d'images, caractéristique du langage généreusement métaphorique du grand poète qu'est Büchner, éclaire d'une lumière significative le mal dont souffre Léonce, cette « difficulté d'être » qui constitue le thème majeur de la pièce.

Georg Büchner

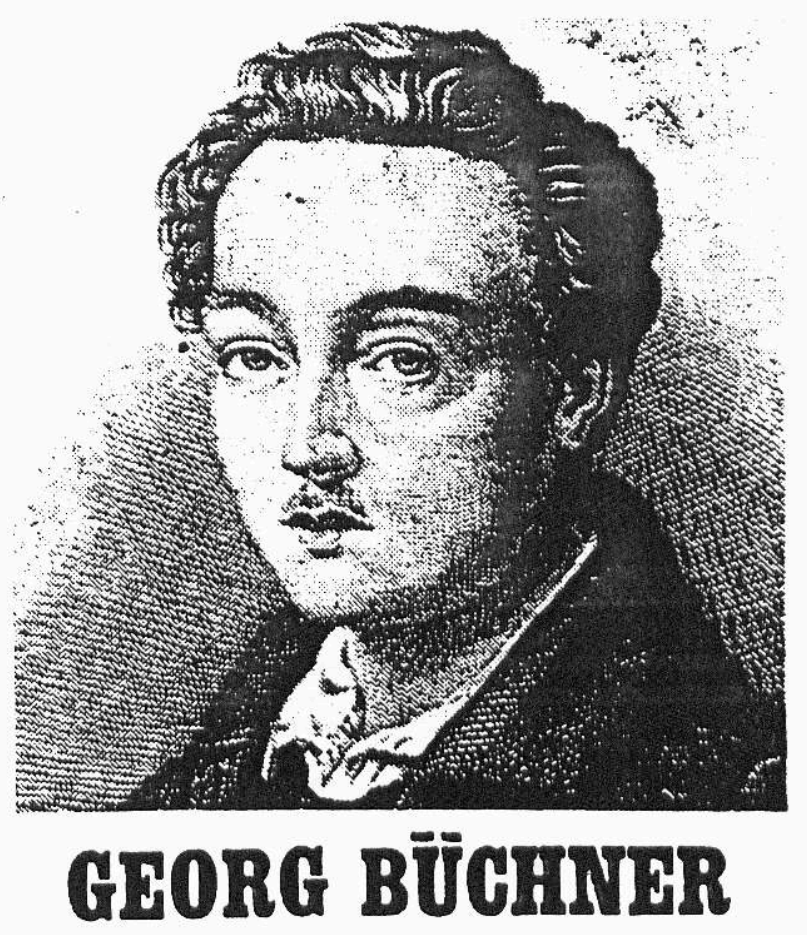


5 D'abord la mort du temps : une paralysie éprouvée en face de l'action, à l'orée d'une existence qu'il s'agit de remplir «comme une grande feuille de papier»; la peur de l'avenir, interminable succession de redites, une perception douloureuse du temps qui semble tourner à vide, ne progresse plus mais procède par répétitions mécaniques. La métaphore de la marionnette commande toute la pièce.

6 La mort du temps et son corollaire, la mort du désir : le sentiment que la fête de la vie est définitivement éteinte et que le silence et l'immobilité de la mort règnent désormais sans partage sur la réalité. Léonce étouffe, il a l'impression d'être enfermé sous une cloche d'air. ${ }^{4}$

7 Les violons sont brisés et il ne reste qu'une immense fatigue. Cette plainte, la pièce la fait entendre à de nombreuses reprises, dans la première phase de l'action surtout, avant que Léonce ne rencontre Léna. Léonce la formule, mais aussi Rosetta, la maîtresse qu'il n'aime pas et dont le chant rejoint le monologue désenchanté de son amant :

Elle danse et chante:

O mes pieds lassés, il vous faut danser

En chaussures bigarrées.

Quand votre désir est de reposer

Au plus secret de la terre!

O mes joues ardentes, il vous faut brûler

Sous de sauvages baisers

Quand vous désirez la blancheur nacrée

De deux roses blanches.

O mes pauvres yeux, il vous faut briller

Dans l'éclat des lustres,

Quand votre désir appelle un sommeil

De douleur dans les ténèbres. ${ }^{5}$

Elle culmine au second acte, au moment où Léonce et Léna se retrouvent. Büchner a mis là en scène une cérémonie étrange, érotique et funèbre à la fois, en plaçant le dialogue amoureux sous le signe d'une sorte d'hymne à la mort :

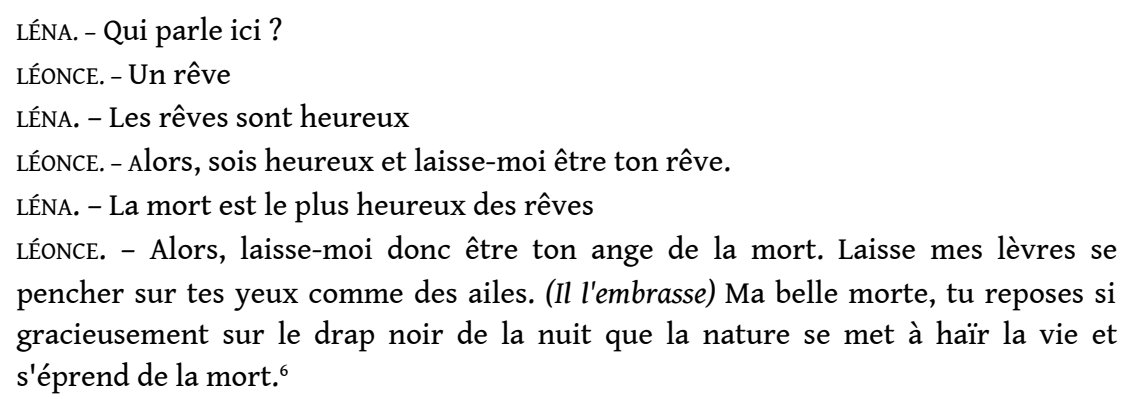

9 Cette surprenante scène d'amour nocturne très voisine de la première scène de La Mort de Danton où Danton fait lui aussi une étrange déclaration à sa femme Julie ${ }^{7}$, est la plus mystérieuse et la plus solennelle de la pièce, la plus poétique peut-être aussi. Elle représente un des sommets de l'œuvre : la comédie s'efface complètement pour donner toute son ampleur au thème central du combat contre l'obsession de la mort. C'est également la plus dramatique parce que Léonce, va tenter de se suicider. C'est en même temps l'instant de la renaissance, car les choses basculent brusquement et le cœur du jeune prince se remet à battre au rythme de la vie.

Ce sont là des aspects majeurs de ce qui ressemble beaucoup à un état dépressif dont on retrouve les accents dans $\mathrm{La}$ Mort de Danton et dans Woyzeck: Ils expriment une sensibilité malheureuse, foncièrement négative, envahie par une conscience 
omniprésente de l'extinction de toute chose, une «lassitude » extrême de l'esprit, que Léna perçoit intimement lorsqu'elle voit Léonce pour la première fois :

Léna et la Gouvernante

LA GOUVERNANTE. - Ne pensez pas à cet homme !

LÉNA. - Il était si vieux sous ses boucles blondes. Le printemps sur les joues et l'hiver dans le cœur! Quel spectacle affligeant. Le corps fatigué trouve son oreiller n'importe où, mais quand l'esprit est las, où peut-il prendre du repos? Il me vient une pensée effroyable: je crois qu'il y a des êtres malheureux, incurablement malheureux du seul fait qu'ils existent. ${ }^{8}$

Büchner a sûrement vécu tout cela. La modernité, la profonde vérité humaine de son témoignage, nous touchent tout autant que la mise en œuvre artistique de sa comédie à travers laquelle il va nous montrer comment ce mal de vivre peut être surmonté.

\section{Et vous Prince, vous n'êtes qu'un livre sans lettres, imprimé uniquement en points de suspension. ${ }^{9}$}

11 Le thème de l'angoisse et de son dépassement constitue en effet l'épine dorsale de la pièce. Il en organise la structure dramatique et la distribution des personnages.

Omniprésent au début de l'œuvre (vivre c'est cracher trois cent soixante-cinq fois de suite sur la même pierre... ${ }^{10}$ ), il est à nouveau là à la fin. Le prince a épousé Léna et la vie recommence. Qu'allons-nous faire maintenant les poches pleines de poupées et de jouets $?^{11}$ A nouveau se dessine l'ombre de la vie morte, vidée par l'habitude, tant redoutée par le jeune Büchner, confronté à la perspective d'une vie bourgeoise «bien rangée » dans la respectabilité et un labeur qui risque d'émousser la ferveur de la vie. Entre ces deux extrémités de la pièce, étrangement voisines, une parenthèse, à la fois dramatique et poétique. Dramatique parce qu'elle structure le déroulement de l'action : pour Léonce, le temps se remet en marche. Il rencontre Léna, oublie la mélancolie et sa paralysie. Poétique car cette alchimie s'opère hors du temps et du quotidien, la nuit, ailleurs, vers l'Italie ou Léonce a fui Léna pour mieux la retrouver.

Ce thème de l'angoisse commande également la distribution des personnages. Büchner nous montre d'une part ceux dont le comportement la justifie, de l'autre ceux qui l'aident à s'en défaire.

Les premiers, les personnages de la pesanteur, sont très schématiques, sans aucune épaisseur psychologique. Ils font office de repoussoirs, chargés de donner des images de l'existence négative, celle que Léonce veut éviter à tout prix, des images de la vie sans la vie, d'êtres myopes, d'automates enfermés dans des certitudes sommaires, satisfaits à peu de frais du confort de l'habitude, des plaisirs du pouvoir : le roi Peter qui règne sur le royaume de Popo (la métaphore est éloquente...), ainsi que la brochette de notables qui vibrionnent dans son sillage.

L'auteur du Messager Hessois ${ }^{12}$ tire à plaisir les fils de ces marionnettes et la comédie y trouve son compte. Sa critique est, nous le verrons, politique, mais elle est aussi philosophique : ces personnages désignent clairement, grands ou petits, les figures d'un jeu de cartes ${ }^{13}$, muettes et figées en dépit de leur agitation, des figures menées par le hasard de la partie. Ils interprètent d'une certaine manière, dans cette pièce apparemment gaie, une danse de mort, indispensable commentaire de la quête de Léonce. Leur présence sur la scène est chargée de nous dire que Léonce et Léna, malgré ce qui peut sembler excessif dans les atermoiements peut-être un peu trop 
complaisants d'un jeune oisif, est une pièce sur l'invention de la vie, une réflexion passionnée sur la façon la plus vivante de vivre.

Ces âmes mortes signalent les écueils du voyage de Léonce, elles s'opposent aux deux personnages qui, eux, vont l'aider dans sa recherche : Valério, l'ami et Léna, l'aimée. Ils sont d'inégale importance.

Léna reste dans l'ombre de Léonce, mais elle a fait, comme lui, le choix de la liberté en refusant le mariage prescrit par l'ordre des choses. Comme lui, elle succombe à l'amour et à sa métamorphose. Elle parle peu, reprend en écho les paroles de Léonce, seul personnage destiné à exposer le débat de la pièce. Le seul avec Valério qui lui, par contre, est le véritable interlocuteur.

17 Valério est indissociable de Léonce. C'est dans le couple qu'ils forment - Büchner y a sans doute incarné des postulations antagonistes qu'il a lui-même vécues : désir de vie, désir de mort - qu'il faut chercher la vérité de l'œuvre. Valério entraîne Léonce hors des sentiers battus. Il est l'artisan du départ, Hermès peut-être, le messager des Dieux ${ }^{14}$. Avec lui, il dit sa peur de l'engourdissement, de l'existence figée et dénonce dans les mêmes termes que lui la marionnette qui guette l'Homme à tout moment, même quand il devient amoureux.

Attention, Messieurs et Dames, ils se trouvent en ce moment à un stade intéressant de leur développement: le mécanisme de l'amour commence à fonctionner, le Monsieur a déjà plusieurs fois porté le châle de la Dame, et la Dame a déjà regardé le ciel plusieurs fois avec des yeux chavirés. Amour, Espérance. Ils paraissent déjà tout à fait accordés, il ne leur manque plus qu'un petit, un minuscule vocable : Amen $!^{15}$

Incontestablement, c'est lui qui mène le jeu. Il est le bon génie chargé de mettre en scène le mariage en effigie du dernier acte : le roi Peter ne veut pas renoncer à la cérémonie prévue et décide de célébrer un mariage factice. Deux mannequins doivent prendre la place du marié et de la mariée. Valério s'arrange pour que les deux mannequins ne soient autres que Léonce et Léna. Au cours de cette cérémonie, ValérioHermès, maître de vie, préside non pas à la mort de l'homme dans la marionnette mais à l'éveil de la marionnette qui devient un être humain. Toute la leçon de la pièce est magistralement résumée dans cette scène parfaitement métaphorique et d'une remarquable efficacité scénique: Büchner fustige les abus de pouvoir, il le prend au piège de ses propres machinations, il exalte la victoire de la vie sur la mort et en même temps, par un rétablissement dramatique extraordinaire, boucle sa comédie.

Valério est donc l'âme de cette aventure où Léonce va trouver remède à ses maux. Il est le maître qui lui indique les périls de sa contemplation fascinée de la mort et de la fuite du temps.

Il l'entraîne, le secoue et le ramène sur terre. Il donne lui-même l'exemple - Büchner nous indique à travers son personnage le goût du bonheur, l'acceptation de la vie et de ses plaisirs, le désir d'être avec les autres et d'œuvrer avec eux - et il critique par ailleurs vigoureusement «le romantisme de lieutenant » de son élève en lui faisant comprendre que son attitude est une impasse et mène à la folie ${ }^{16}$. Ce n'est pas un hasard si c'est lui qui empêche Léonce de se tuer après la rencontre avec Léna. Il montre bien le chemin de la vie. 


\section{La société humaine} réaliste où Büchner, comme il le fait dans Woyzeck, dénonce avec vigueur les injustices de la société allemande de son époque. Une société qu'il a analysée avec une remarquable lucidité, qu'il a essayé de transformer et qui lui a bien rendu la monnaie de sa pièce. Il faut tendre l'oreille aux discours apparemment dérisoires des maîtres et des valets. Büchner se borne à suggérer, mais les coups portés sont d'une redoutable précision.

21 Les maitres. Ces tyranneaux allemands exerçant un pouvoir absolu sur de minuscules principautés: l'arbitraire, l'autoritarisme, les dépenses somptueuses, l'égoïsme des souverains, indifférents à la misère de leurs sujets, les courtisans serviles, la bêtise surtout. Une bourgeoisie de notables, à l'ombre du pouvoir, prétendant constituer « la société humaine ».

(Sur un ton nasillard:)

Vous voyez ici, Messieurs et Mesdames, deux personnages de sexe différent, un mâle et une femelle, un monsieur et une dame. Rien qu'artifice et mécanique, rien que du carton et des ressorts d'horlogerie! Chacun porte un ressort très fin, très fin, sous l'ongle du petit orteil du pied droit, on appuie légèrement, et la mécanique fonctionne bel et bien pendant cinquante ans. Ces personnages sont fabriqués avec un tel art que si l'on ne sait pas qu'ils sont de simples créatures de carton, on ne peut absolument pas les distinguer des autres hommes. Rien ne s'oppose à ce qu'on en fasse des membres de la société humaine. Ils sont d'une grande noblesse, puisqu'ils parlent le haut allemand. Ils sont très moraux, puisqu'ils se lèvent au coup de cloche, prennent leur repas de midi au coup de cloche, et se mettent au lit au coup de cloche. Ils ont également une bonne digestion, ce qui prouve qu'ils ont une bonne conscience. Ils sont doués d'une pudeur très délicate, car la Dame n'a point de mot pour l'idée de pantalon et il est absolument impossible au Monsieur de monter un escalier derrière une femme ou de passer devant elle. Ils sont très cultivés; la Dame chante tous les opéras nouveaux et le Monsieur porte des manchettes. ${ }^{17}$

C'est Valério qui parle mais c'est Büchner qui montre du doigt une classe sociale qu'il connaît pour l'avoir vue de près : engoncée dans la respectabilité des bonnes manières et de la culture.

Les valets. Des paysans misérables, la faim, le courage, la vie subie sans renâcler, des pauvres, obligés d'applaudir au passage du Seigneur qui les tient durement en laisse :

LE MAiTRE D'ECOLE. - Ils se tiennent si bien au milieu de leurs souffrances qu'ils se soutiennent les uns les autres depuis pas mal de temps. Ils se versent bravement de l'alcool dans le corps, faute de quoi ils ne pourraient pas tenir aussi longtemps à la chaleur.

LE CONSEILLER PRovincial. - Attention, vous autres, il est dit dans le programme : "Tous les sujets seront vêtus de vêtements propres, ils seront bien nourris et disposés avec des visages heureux tout le long de la route». Ne nous faites pas honte !18

Toute la révolte de Büchner contre l'iniquité, la sympathie qu'il éprouve à l'égard des démunis du Grand Duché de Hesse, si perceptibles dans Woyzeck mais aussi dans La Mort de Danton, quand il évoque le désarroi du peuple de Paris en 1794, parlent dans ces croquis acérés. 
dit aussi son désir de changer l'ordre des choses. Il suggère clairement, au-delà de la féerie et de la comédie, une autre répartition des cartes : inverser les rôles, chasser le roi Peter de son royaume de Popo, redonner leur dignité à ses sujets en les délivrant de l'oppression et d'un labeur qui les usent et découragent en eux le désir de vie, donner le pouvoir à des hommes comme Léonce et Valério, épris de liberté. Au bout du compte, le retour de Léonce sur le trône au terme de son voyage, avec Valério comme ministre, exprime peut-être la pensée profonde de cette pièce qui est également une pièce subversive :

LÉONCE. - Mais je sais bien mieux que toi ce que tu veux : nous allons faire démolir toutes les pendules et interdire tous les calendriers, nous compterons les heures et les mois d'après la seule horloge des fleurs, des boutons et des fruits. Puis nous ferons entourer notre pays de miroirs ardents, afin qu'il n'y ait plus d'hiver, et que nous puissions nous distiller en été jusqu'à Ischia et Capri, et que nous nous cachions toute l'année parmi les violettes et les roses, les orangers et les lauriers.

VALÉRIo. - Et moi, je deviens Ministre d'Etat; on promulguera un décret suivant lequel toute personne qui aura des ampoules aux mains sera placée sous tutelle; toute personne qui tombera malade à force de travail sera justiciable d'un tribunal criminel, et tous ceux qui se vantent de manger leur pain à la sueur de leur front seront déclarés déments et nuisibles à la société; puis nous nous étendrons à l'ombre et nous prierons Dieu de nous envoyer des macaronis, des melons et des figues, des gorges mélodieuses, des corps classiques et une religion commode. ${ }^{19}$

Non pas le pays de Cocagne, assurément, mais une utopie. Derrière le clin d'œil malicieux de l'auteur, révolutionnaire en mal de révolution, l'utopie de la cité idéale où l'être humain aurait le droit et le loisir de s'abandonner à ce qui est - pourquoi pas ? nous dit Büchner - sa vocation : au bonheur d'être sur la terre, avec d'autres hommes, en compagnie des fleurs et des fruits.

\section{NOTES}

1. Les sources littéraires suivantes sont repérables dans Léonce et Léna : la Bible; la Tradition de la Comédia del'arte; Brentano; Goethe (Werther, Faust); Heine (Die Harzreise); Jean-Paul; E.T.A. Hoffman ; Kant ; Klinger ; Lenz ; Schiller ; F. Schlegel (Lucinde) ; Tieck ; Shakespeare (Hamlet, Comme il vous plaira, Le Marchand de Venise, Henri IV) ; Alfred de Musset (Fantasio, On ne badine pas avec l'amour).

2. Büchner sera poursuivi par la justice de son pays, le Grand Duché de Hesse, pour avoir eu des activités subversives. Il échappera de peu à l'arrestation et mourra en exil, en Suisse, peu de temps après. (cf. les repères chronologiques en annexe).

3. Georg Büchner, Léonce et Léna, texte français de Marthe Robert, L'Arche, 1983, p. 108.

4. Léonce et Léna, op. cit., p. 108.

5. Léonce et Léna, op. cit., p. 106.

6. Léonce et Léna, op. cit., p. 124.

7. DANTON. - Julie, tu m'es chère comme le tombeau.

JULIE, se détournant. - Oh!

DANTON. - Non! Écoute, les gens prétendent que c'est la paix qui règne dans le tombeau, que la tombe et le repos ne sont qu'une seule et même chose. S'il en est ainsi, couché sur ton sein, je gis 
déjà sous la terre. Ma douce tombe! Ta voix est un glas funèbre et ton cœur, mon cercueil.

Georg Büchner, La Mort de Danton, L'Arche, 1953, p. 16.

8. Léonce et Léna, op. cit., p. 123.

9. Léonce et Léna, op. cit., p. 112.

10. «Voyez-vous j'ai d'abord à cracher sur cette pierre, trois cent soixante-cinq fois de suite. Avez-vous déjà essayé ? Faites-le. C'est un passe-temps original. Et puis... voyez-vous cette poignée de sable ? (Il prend du sable, le jette en l'air et le rattrape avec le dos de la main). Maintenant je la lance en l'air. Voulez-vous parier? Combien de grains ai-je maintenant sur le dos de la main? Pair ou impair?»

Léonce et Léna, op. cit., p. 99.

11. « Eh bien Léna, tu vois maintenant comme nous avons les poches pleines, pleines de poupées et de jouets? Qu'allons-nous en faire?»

Léonce et Léna, op. cit., p. 136.

12. Le Messager Hessois est le titre du pamphlet incendiaire que Büchner écrivit pour dénoncer les injustices en Hesse et qui lui valut des poursuites judiciaires et l'exil (cf. repères chronologiques). 13. La métaphore du jeu de cartes, pour Büchner à la fois le symbole de la vanité de la comédie sociale, de la vie amoindrie et de la toute-puissance du hasard, rejoint celle de la marionnette.

« En bas, la terre et l'eau sont comme une table où le vin se répand et nous sommes posés dessus comme des cartes à jouer avec lesquelles Dieu et le Diable feraient une partie pour passer le temps; vous êtes le Roi et je suis le Valet, il ne manque que la Dame, une belle dame, avec un grand cœur de pain d'épice sur la poitrine et une énorme tulipe où elle enfouirait sentimentalement son long nez. »

Léonce et Léna, op. cit., p. 121.

De même dans La Mort de Danton:

"J'ourdissais une intrigue avec une reine de cartes, mes doigts étaient des princes métamorphosés en araignées. Vous madame, étiez la fée, mais les choses se gâtèrent, la dame accouchait à chaque instant d'un valet. Je ne permettais pas de semblables jeux à ma fille. Les rois tombent sur les reines sans pudeur et les valets sortent aussitôt. »

La Mort de Danton, op. cit., p. 16.

14. Léonce quand il fait la connaissance de Valério y fait allusion :

LÉONCE avec un enthousiasme comique. - Viens sur mon cœur ! Es-tu l'un de ces divins qui avancent sans effort sur la route de la vie et gardent le front pur à travers sueurs et poussières, semblables aux dieux bienheureux qui entrent dans l'olympe avec des semelles étincelantes et des corps florissants? Viens, viens !

Léonce et Léna, op. cit., p. 102.

15. Léonce et Léna, op. cit., p. 134.

16. VALÉRIO. - Non, le chemin qui mène à l'asile d'aliénés n'est pas si long. Il est facile à trouver, je connais tous les sentiers, tous les chemins vicinaux, toutes les routes y conduisent. Je l'imagine déjà, cheminant sur une large allée par un jour d'hiver glacé, le chapeau sous le bras, il cherche l'ombre sous les arbres dépouillés et s'évente avec son mouchoir. Il est fou !

Léonce et Léna, op. cit., p. 123.

17. Léonce et Léna, op. cit., p. 133.

18. Léonce et Léna, op. cit., p. 128.

19. Léonce et Léna, op. cit., p. 136. 


\section{AUTEURS}

GÉRARD JUGAN

Maître de conférences de Littérature allemande à l'Université de Franche-Comté, il est l'auteur de plusieurs articles sur le théâtre allemand. 